Punjab University Journal of Mathematics (2021),53(9),753-763

https://doi.org/10.52280/pujm.2021.531005

\title{
Oscillation and nonoscillation criteria for second order difference equations with generalized difference operators
}

\author{
Nurettin DO GAN \\ Department of Computer Engineering, \\ Faculty of Technology, \\ Selçuk University, Konya / TURKEY, \\ Email: ndogan@ymail.com \\ Yaşar BOLAT \\ Department of Mathematics, \\ Faculty of Science and Arts, \\ Kastamonu University, 37100-Kastamonu/TURKEY, \\ Email: ybolat@kastamonu.edu.tr
}

Received: 15 February, 2021 / Accepted: 27 August, 2021 / Published online: 27 October, 2021

\begin{abstract}
In this study we investigate some new oscillation and nonoscillation criteria and generalize and improve some results in the literatures for second order nonlinear difference equation with generalized difference operators of the form

$$
\Delta_{l, a}\left(p_{n} \Delta_{l, a} x_{n}\right)+q_{n}\left(\Delta_{l, a} x_{n}\right)^{\beta}=F\left(n, x_{n}, \Delta_{l, b} x_{n}\right),
$$
\end{abstract}

where $\Delta_{l, \sigma}$ is generalized difference operator such that defined as $\Delta_{l, \sigma} x_{n}=$

included. $x_{n+l}-\sigma x_{n}$, and $F: \mathbb{N} \times \mathbb{R}^{2} \rightarrow \dot{R}$. Also, some examples illustrating the results are

AMS (MOS) Subject Classification Codes: 39A10; 39A21

Key Words: Oscillation; nonoscillation; difference equation; generalized difference equation; generalized difference operator.

\section{INTRODUCTION}

Consider the generalized difference equation of the form

$$
\Delta_{l, a}\left(p_{n} \Delta_{l, a} x_{n}\right)+q_{n}\left(\Delta_{l, a} x_{n}\right)^{\beta}=F\left(n, x_{n}, \Delta_{l, b} x_{n}\right), \quad n \in \mathbb{N},
$$

where $\mathbb{N}=\{1,2, \cdots\}, a(\neq 0)$ and $b$ are real numbers, $l \in \mathbb{N}, \beta \in \mathbb{R}^{+}, \Delta_{l, a}$ is generalized difference operator that has the property of $\Delta_{l, a}^{k} x_{n}=\Delta_{l, a}\left(\Delta_{l, a}^{k-1} x_{n}\right)$ and defined $\Delta_{l, a} x_{n}=x_{n+l}-a x_{n},\left\{p_{n}\right\}$ and $\left\{q_{n}\right\}$ are real sequences, $F: \mathbb{N} \times \mathbb{R}^{2} \rightarrow \mathbb{R}, x: \mathbb{N} \rightarrow \mathbb{R}$, $x_{n}=x(n)$ for $n \in \mathbb{N}$. The cases of eventually positive, negative and oscillatory properties of sequences $\left\{p_{n}\right\}$ and $\left\{q_{n}\right\}$ are considered for all $n \in \mathbb{N}$ in some of the results. 
By a solution of ( 1.1 ), we mean a real sequence $\left\{x_{n}\right\}$ satisfying ( 1.1 ) for all $n \in \mathbb{N}$. A solution $\left\{x_{n}\right\}$ of $(1.1)$ is said to be oscillatory if $x_{s} x_{s+l} \leq 0$ for $s \in \mathbb{N}_{i}=$ $\{i, i+l, i+2 l, \ldots\}$, where $i \in \mathbb{N}$. In other words, a solution is said to be oscillatory if it is neither eventually positive nor eventually negative. Otherwise it is called nonoscillatory. The difference equation is called oscillatory if all its solutions are oscillatory.

Difference and differential equations find numerous applications in natural science and technology $[12,14,15]$. For instance, they are frequently used for the study of population dynamics and stability theory [18, 36], synchronization analysis [20, 21, 37], circuit theory $[4,22,23]$ and so on. Therefore, those equations have been of a great interest during the last few decades. The problem of determining oscillation and nonoscillation of solutions of difference and differential equations has been a very active area of research in the last years $[9,10,11,13,17,26,6]$, and for some results on the oscillation and nonoscillation topics we refer to the monographs of Agarwal, Grace and O'Regan [1], Agarwal [2] and Agarwal and Wong [3].

Following some second order linear and nonlinear difference equations when $a=b=$ $l=1$ are investigated by some authors and obtained some oscillation and nonoscillation criteria for these equations. For examples; second order linear difference equation of the form

$$
\Delta^{2} x_{n-1}+q_{n} x_{n}=0, n \in \mathbb{N},
$$

is investigated by Cheng at all. [16] and Zhang and Zhou [35]. The more general case of the above second order difference equation of the form

$$
\Delta^{2} x_{n-1}+q_{n} f\left(x_{n}\right)=0, n \in \mathbb{N},
$$

is considered by Zhang and Chen [34]. He [19] studied the second order nonlinear difference equation of the form

$$
\Delta\left(r_{n} \Delta x_{n}\right)+f\left(n, x_{n}\right)=0, \quad n \geq 0 .
$$

Chen and Erbe [7] and Chen [8] invesigated the second order advanced linear difference equation of the form

$$
\Delta\left(r_{n} \Delta x_{n}\right)+p_{n} x_{n+1}=0, \quad n \geq 0 .
$$

More genaral case of this last second order advanced nonlinear difference equation of the form

$$
\Delta\left(c_{n} \Delta x_{n}\right)+p_{n} x_{n+1}^{\gamma}=0, \quad n \geq 0,
$$

is considered by Zhang [33]. Szafranski and Szmanda [27] studied the more general second order nonlinear difference equations of the forms

$$
\Delta^{2} x_{n}+f\left(n, x_{n}, \Delta x_{n}\right)=0, n \geq 0,
$$

and

$$
\Delta^{2} x_{n}+a_{n} f\left(n, x_{n}, \Delta x_{n}\right)=0, n \geq 0 .
$$

More general case of these above second order advanced damped difference equation of the form

$$
\Delta^{2} x_{n}+p_{n} \Delta x_{n}+q_{n} f\left(x_{n+1}\right)=0, n \geq 0,
$$

is considered by Thandapani and Lalli [30]. Thandapani [31] studied the more general case of above advanced damped nonlinear difference equation of the form

$$
\Delta\left(a_{n}\left(\Delta x_{n}\right)\right)+p_{n} \Delta x_{n}+q_{n} f\left(x_{n+1}\right)=0, n \geq 0 .
$$


Wong and Agarwal [32] considered second order nonlinear difference equation of the form

$$
\Delta\left(a_{n}\left(\Delta x_{n}\right)^{\sigma}\right)+b_{n}\left(\Delta x_{n}\right)^{\sigma}+F\left(n, x_{n}, \Delta x_{n}\right)=0, \quad n \geq 0,
$$

and Szmanda [28] investigated the more general case of above nonlinear difference equation of the form

$$
\Delta\left(r_{n} \Delta x_{n}\right)+a_{n} f\left(n, x_{n}, \Delta x_{n}\right)=g\left(n, x_{n}, \Delta x_{n}\right), \quad n \in \mathbb{N} .
$$

However, it seems that there are scarce study concerning the oscillatory and nonoscillatory behaviour of solutions of second order difference equations with generalized difference operators. For examples; In the beginning, Parhi [24] invesigated oscillation and nonoscillation behavior for second order difference equations with generalized difference operators of the forms

and

$$
\Delta_{a}\left(p_{n-1} \Delta_{a} x_{n-1}\right)+q_{n} x_{n}=0
$$

$$
\Delta_{a}\left(p_{n-1} \Delta_{a} x_{n-1}\right)+q_{n} x_{n}=f(n)
$$

where $\Delta_{a}$ is generalized difference operator and defined $\Delta_{a} x_{n}=x_{n}-a x_{n}$. In [25], Popenda studied the oscillatory and nonoscillatory behaviour of solutions of difference equation involving generalized difference operators of the form

$$
\Delta_{a}^{2} x_{n}=F\left(n, x_{n}, \Delta_{b} x_{n}\right), \quad n \in \mathbb{N},
$$

when $l=1, p_{n}=1$ and $q_{n}=0$ in ( 1. 1 ). Tan and Yang [29] discussed the oscillatory and nonoscillatory behaviour of solutions of difference equation with generalized difference operators of the form

$$
\Delta_{a}\left(p_{n} \Delta_{a} x_{n}\right)+q_{n} \Delta_{a} x_{n}=F\left(n, x_{n}, \Delta_{b} x_{n}\right), \quad n \in \mathbb{N},
$$

when $l=1, \beta=1$ and $p_{n}$ and $q_{n}$ are real sequences in (1.1). Bolat and Akın [5] investigated the oscillatory behaviour of solutions of $m$ - order difference equations with generalized difference operator of the form

$$
\Delta_{b}\left(p_{n}\left(\Delta_{b}^{m-1} x_{n}\right)^{\alpha}\right)+q_{n} x_{n-\sigma}^{\beta}=0, n \in \mathbb{N} .
$$

Motivated by these observation, our aim in this study is to obtain sufficient conditions for the oscillation and nonoscillation of nontrivial solutions of ( 1.1 ) without the usual restrictive conditions on the coefficients sequences $p, q$ and function $F$. Moreover, some examples illustrating the results are included.

Throughout the study we denote by $X$ the set of nontrivial solutions of ( 1.1 ) and define $X_{1}=\left\{x \in X: \Delta_{l, a} x_{k}=0\right.$ holds for some $\left.k \in \mathbb{N}\right\}$ and $X_{2}=X \backslash X_{1}$.

\section{Main Results}

\subsection{Oscillatory theorems.}

Theorem 1. Let $a<0,0<\beta<1$ such that $\frac{2}{\beta-1}$ is the ratio of two odd integers, $p_{n}>0$, $q_{n}>0$ and the condition

$$
\left(C_{1}\right)\left\{\begin{array}{c}
F(n, u, v)=0, \quad v+(b-a) u=0, \quad n \in \mathbb{N}, u, v \in \mathbb{R}, \\
\frac{v+(b-a) u}{p_{n+l}} F(n, u, v) \leq 0, \quad v+(b-a) u \neq 0, \quad n \in \mathbb{N}, u, v \in \mathbb{R}
\end{array} \quad\right. \text { is sat- }
$$

isfied, then all nontrival solutions of (1.1) are oscillatory. 
Proof. Let $x$ be any nontrivial solutions of (1.1). Then $x \in X_{1}$ or $x \in X_{2}$.

(i) If $x \in X_{1}$, then there is some $k \in \mathbb{N}$ such that $\Delta_{l, a} x_{k}=x_{k+l}-a x_{k}=0$. Therefore considering $F\left(k, x_{k}, \Delta_{l, b} x_{k}\right)=0$ for some $k \in \mathbb{N}$ according to $\left(C_{1}\right)$ and letting $n=k$ in $(1.1)$, we reach $\Delta_{l, a}\left(p_{k} \Delta_{l, a} x_{k}\right)=0$. Hence we obtain $\Delta_{l, a} x_{k+l}=\frac{a p_{k}}{p_{k+l}} \Delta_{l, a} x_{k}=$ 0 . By reapiting this same process, we obtain $\Delta_{l, a} x_{k+2 l}=0$. By induction, we reach $\Delta_{l, a} x_{k+j l}=0$ for some $j \in \mathbb{N}$. Therefore we have

$$
x_{k+j l}=a^{j} x_{k} \text { for some } j \in \mathbb{N}_{0} .
$$

Since $a<0$ and $x_{k} \neq 0$, we have $x_{k+j l} x_{k+(j+1) l}=a^{2 j+1}\left(x_{k}\right)^{2}<0$ for some $j \in \mathbb{N}_{0}$. Hence $x$ is oscillatory.

(ii) If $x \in X_{2}$, then $\Delta_{l, a} x_{k} \neq 0$. If we show that $x$ is oscillatory, then all $x \in X$ are oscillatory. Hence we reach our aim. Now, suppose that $x$ is nonoscillatory. Then it is either eventually positive or eventually negative.

Without loss of general assume that $x$ is eventually positive (When $x$ is eventually negative can be proved in similar manner), then we can find an even integer $m$ such that $x_{n}>0$ for $n \geq m$. We can rewrite the (1.1) in the form

$$
\Delta_{l, a} x_{n+l}=\frac{a p_{n}}{p_{n+l}} \Delta_{l, a} x_{n}+\frac{q_{n}}{p_{n+l}}\left(\Delta_{l, a} x_{n}\right)^{\beta}+\frac{1}{p_{n+l}} F\left(n, x_{n}, \Delta_{l, b} x_{n}\right), \quad n \in \mathbb{N} .
$$

Letting $n=m$ in (2. 2 ) and multiplying the both sides by $\Delta_{l, a} x_{m}$, we derive

$$
\Delta_{l, a} x_{m} \Delta_{l, a} x_{m+l}=\frac{a p_{m}}{p_{m+l}}\left(\Delta_{l, a} x_{m}\right)^{2}-\frac{q_{m}}{p_{m+l}}\left(\Delta_{l, a} x_{m}\right)^{\beta+1}+\frac{\Delta_{l, a} x_{m}}{p_{m+l}} F\left(m, x_{m}, \Delta_{l, b} x_{m}\right)
$$

for some $m \in \mathbb{N}$. Setting $G\left(Y_{m}\right)=A_{m} Y_{m}^{2}-B_{m} Y_{m}^{\beta+1}$ in (2.3), where $A_{m}=\frac{a p_{m}}{p_{m+l}}$, $B_{m}=\frac{q_{m}}{p_{m+l}}$ and $Y_{m}=\Delta_{l, a} x_{m}$, we obain

$$
G_{\max }=\left(\frac{\beta-1}{\beta+1}\right) A_{m}\left(\frac{2 A_{m}}{(\beta+1) B_{m}}\right)^{\frac{2}{\beta-1}}<0
$$

at the point $Y_{m}=\left(\frac{2 A_{m}}{(\beta+1) B_{m}}\right)^{\frac{1}{\beta-1}}$. Therefore considering $\left(C_{1}\right)$ from ( 2. 3 ) and ( 2. 4 ) we have

$$
\begin{aligned}
\Delta_{l, a} x_{m} \Delta_{l, a} x_{m+l} & \leq\left(\frac{\beta-1}{\beta+1}\right) A_{m}\left(\frac{2 A_{m}}{(\beta+1) B_{m}}\right)^{\frac{2}{\beta-1}} \\
& +\frac{\left(\Delta_{l, b} x_{m}+(b-a) x_{m}\right)}{p_{m+l}} F\left(m, x_{m}, \Delta_{l, b} x_{m}\right) \\
& \leq 0 .
\end{aligned}
$$

Since $x \in X_{2}$ and $\Delta_{l, a} x_{m}=x_{m+l}-a x_{m}>0$, by (2.5) we have

$$
\Delta_{l, a} x_{m+l}=x_{m+2 l}-a x_{m+l}<0
$$

or

Hence we obtain

$$
x_{m+2 l}<a x_{m+l}<0 .
$$

$$
x_{m+l} x_{m+2 l}<0 .
$$


Repeating the same process from ( 2. 3 ) - ( 2. 8 ), we reach $x_{m+j l} x_{m+(j+1) l}<0$ for all $j, m \in \mathbb{N}$. This contradicts with to be $x$ is eventually positive. Hence the proof is completed.

Theorem 2. Let a $<0,0<\beta<1$ such that $\frac{2}{\beta-1}$ is the ratio of two odd integers, $p_{n}<0$, $q_{n}<0$ and the condition $\left(C_{1}\right)$ is satisfied, then all nontrival solutions of ( 1.1$)$ are oscillatory.

Proof. The proof can be proved as in Theorem 1. Therefore we omitted it in here.

Theorem 3. Let $a<0, \beta>1$ such that $\frac{2}{\beta-1}$ is the ratio of two odd integers, $p_{n}$ and $q_{n}$ are oscillatory with $p_{n} p_{n+l}<0, p_{n} q_{n}>0$ and the condition

$$
\left(C_{2}\right)\left\{\begin{array}{cc}
F(n, u, v)=0, & v+(b-a) u=0, \\
\left(\frac{\beta-1}{\beta+1}\right) \frac{a p_{n}}{p_{n+l}}\left(\frac{2 a p_{n}}{(\beta+1) q_{n}}\right)^{\frac{2}{\beta-1}}+\frac{v+(b-a) u}{p_{n+l}} F(n, u, v) \leq 0, v+(b-a) u \neq 0,
\end{array}\right.
$$

where $n \in \mathbb{N}, u, v \in \mathbb{R}$, is satisfied, then all nontrival solutions of (1.1) are oscillatory.

Proof. As in Theorem 1 can be proved. Therefore we omitted it in here.

Remark 1. If $a=b$, then conditions $\left(C_{1}\right)$ and $\left(C_{2}\right)$ reduce to

$\left(C_{1}^{*}\right) \frac{v}{p_{n+l}} F(n, u, v) \leq 0$ for $v \neq 0, n \in \mathbb{N}, u, v \in \mathbb{R}$ and

$\left(C_{2}^{*}\right) \quad\left(\frac{\beta-1}{\beta+1}\right) \frac{a p_{n}}{p_{n+l}}\left(\frac{2 a p_{n}}{(\beta+1) q_{n}}\right)^{\frac{2}{\beta-1}}+\frac{v}{p_{n+l}} F(n, u, v) \leq 0$ for $v \neq 0, n \in \mathbb{N}, u, v \in \mathbb{R}$ respectively.

Remark 2. If $b=0$, then ( 1.1 ) becomes the generalized difference equation of the form

$$
\Delta_{l, a}\left(p_{n} \Delta_{l, a} x_{n}\right)+q_{n}\left(\Delta_{l, a} x_{n}\right)^{\beta}=F\left(n, x_{n}, x_{n+l}\right), \quad n \in \mathbb{N},
$$

and for this equation conditions $\left(C_{1}\right)$ and $\left(C_{2}\right)$ reduce to

$$
\begin{aligned}
& \left(C_{1}^{* *}\right) \frac{\left(v^{*}-a u\right)}{p_{n+l}} F\left(n, u, v^{*}\right) \leq 0 \text { for }\left(v^{*}-a u\right) \neq 0, n \in \mathbb{N}, u, v^{*} \in \mathbb{R} \text { and } \\
& \left(C_{2}^{* *}\right)\left(\frac{\beta-1}{\beta+1}\right) \frac{a p_{n}}{p_{n+l}}\left(\frac{2 a p_{n}}{(\beta+1) q_{n}}\right)^{\frac{2}{\beta-1}}+\frac{\left(v^{*}-a u\right)}{p_{n+l}} F\left(n, u, v^{*}\right) \leq 0 \text { for }\left(v^{*}-a u\right) \neq 0, n \in \mathbb{N}, \\
& u, v^{*} \in \mathbb{R} \text { respectively. }
\end{aligned}
$$

\subsection{Some examples to oscillatory equations.}

Example 1. Consider second order difference equation with generalized difference operator of the form

$$
\Delta_{3,-\frac{7}{8}}^{2} x_{n}+\frac{1}{64}\left(\Delta_{3,-\frac{7}{8}} x_{n}\right)^{\frac{1}{3}}=\frac{161}{128} x_{n}+\frac{5}{256}\left(\Delta_{3,3} x_{n}\right)^{3},
$$

where $a=-\frac{7}{8}, b=3, l=3, p_{n}=1, q_{n}=\frac{1}{64}, \beta=\frac{1}{3}, F\left(n, x_{n}, \Delta_{3,3} x_{n}\right)=\frac{161}{128} x_{n}+$ $\frac{5}{256}\left(\Delta_{3,3} x_{n}\right)^{3}$. Since

$$
\begin{aligned}
\frac{v+(b-a) u}{p_{n+l}} F(n, u, v) & =\Delta_{3,-\frac{7}{8}}(-1)^{n}\left(\frac{161}{128}(-1)^{n}+\frac{5}{256}\left(\Delta_{3,3}(-1)^{n}\right)^{3}\right) \\
& =-\frac{1}{1024}<0
\end{aligned}
$$


is satisfied for $v+(b-a) u=\Delta_{3,-\frac{7}{8}} x_{n}=\Delta_{3,-\frac{7}{8}}(-1)^{n}=-\frac{1}{8}(-1)^{n} \neq 0$, the second part of condition $\left(C_{1}\right)$ and all assumptions of Theorem 1 are satisfied. Then every solution of equation (2.9) is oscillatory. So one of solutions is $x_{n}=(-1)^{n}$.

Example 2. Consider second order difference equation with generalized difference operators of the form

$$
\left(\Delta_{3,-1}\left(p_{n} \Delta_{3,-1} x_{n}\right)\right)+q_{n}\left(\Delta_{3,-1} x_{n}\right)^{\frac{1}{3}}=3 x_{n}+\Delta_{3,2} x_{n},
$$

where $a=-1, l=3, p_{n}, q_{n}$ are any real sequences, $\beta=\frac{1}{3}, F\left(n, x_{n}, \Delta_{3,2} x_{n}\right)=$ $3 x_{n}+\Delta_{3,2} x_{n}$. Therefore the first part of condition $\left(C_{1}\right)$ and all assumptions of theorem 1 are satisfied. Then every solution of equation (2.10) is oscillatory. So one of solutions is $x_{n}=(-1)^{n}$. So indeed the first part of condition $\left(C_{1}\right)$

$$
\begin{aligned}
F\left(n, x_{n}, \Delta_{3,2} x_{n}\right) & =3 x_{n}+\Delta_{3,2} x_{n} \\
& =3(-1)^{n}+\Delta_{3,2}(-1)^{n} \\
& =3(-1)^{n}-3(-1)^{n}=0
\end{aligned}
$$

is satisfied for $v+(b-a) u=\Delta_{3,-1} x_{n}=\Delta_{3,-1}(-1)^{n}=0$.

Example 3. Consider second order difference equation with generalized difference operators of the form

$$
\left(\Delta_{3,-\frac{1}{3}}\left(-2 \Delta_{3,-\frac{1}{3}} x_{n}\right)\right)-\left(\frac{2}{3}\right)^{\frac{2}{5}}\left(\Delta_{3,-\frac{1}{3}} x_{n}\right)^{\frac{3}{5}}=\frac{7}{9} x_{n}+\frac{2}{3} \Delta_{3, \frac{1}{2}} x_{n},
$$

where $a=-\frac{1}{3}, l=3, p_{n}=-2, q_{n}=-\left(\frac{2}{3}\right)^{\frac{2}{5}}, \beta=\frac{3}{5}, F\left(n, x_{n}, \Delta_{3, \frac{1}{2}} x_{n}\right)=\frac{7}{9} x_{n}+$ $\frac{2}{3} \Delta_{3, \frac{1}{2}} x_{n}$. Therefore the second part of condition $\left(C_{1}\right)$ and all assumptions of theorem 2 are satisfied. Then every solution of equation (2.11) is oscillatory. So one of solutions is $x_{n}=(-1)^{n}$. So indeed the second part of condition $\left(C_{1}\right)$

$$
\begin{aligned}
\frac{v+(b-a) u}{p_{n+l}} F(n, u, v) & =\frac{\Delta_{3,-\frac{1}{3}}(-1)^{n}}{-2}\left(\frac{7}{9}(-1)^{n}+\frac{2}{3} \Delta_{3, \frac{1}{2}}(-1)^{n}\right) \\
& =-\frac{2}{27}<0,
\end{aligned}
$$

is satisfied for $v+(b-a) u=\Delta_{3,-\frac{1}{3}} x_{n}=\Delta_{3,-\frac{1}{3}}(-1)^{n}=-\frac{2}{3}(-1)^{n} \neq 0$.

Example 4. Consider second order difference equation with generalized difference operator of the form

$$
\Delta_{3,-\frac{1}{2}}\left((-2)^{n} \Delta_{3,-\frac{1}{2}} x_{n}\right)+\left(-\frac{1}{2}\right)^{n}\left(\Delta_{3,-\frac{1}{2}} x_{n}\right)^{\frac{1}{3}}=-\frac{17.2^{n}}{4}\left(x_{n}\right)^{2}-\frac{1}{9.2^{\frac{1}{3}+n}}\left(\Delta_{3,2} x_{n}\right)^{2},
$$

wehere $a=-\frac{1}{2}, l=3, p_{n}=(-2)^{n}, q_{n}=\left(-\frac{1}{2}\right)^{n}, \beta=\frac{1}{3}, F\left(n, x_{n}, \Delta_{3,2} x_{n}\right)=$ $-\frac{17}{4} 2^{n}\left(x_{n}\right)^{2}-\frac{1}{2^{\frac{1}{3}+n_{9}}}\left(\Delta_{3,2} x_{n}\right)^{2}$. Therefore the second part of condition $\left(C_{2}\right)$ and all assumptions of theorem 3 are satisfied. Then every solution of equation ( 2. 12 ) is oscillatory. So one of solutions is $x_{n}=(-1)^{n}$. So indeed the second part of condition 
$\left(C_{2}\right)$

$$
\begin{aligned}
\left(\frac{\beta-1}{\beta+1}\right) \frac{a p_{n}}{p_{n+l}}\left(\frac{2 a p_{n}}{(\beta+1) q_{n}}\right)^{\frac{2}{\beta-1}}+\frac{v+(b-a) u}{p_{n+l}} F(n, u, v) & =\frac{1}{864.4^{3(n-1)}}-\frac{17}{64} \\
& -\frac{1}{2^{2 n+\frac{13}{3}}} \\
& <0, \quad n \geq 1,
\end{aligned}
$$

is satisfied for $v+(b-a) u=\Delta_{3,-\frac{1}{2}} x_{n}=\Delta_{3,-\frac{1}{2}}(-1)^{n}=-\frac{1}{2}(-1)^{n} \neq 0$.

Remark 3. Examples in $1,2,3$ and 4 if $l=0, b=0, F(n, u, v)=0 \quad($ or $F(n, u, v)=$ $\left.\lambda(I f-1)^{n}\right)$ are taken, it is seen that the results of Parhi, Popenda and Yang are true.

\subsection{Nonoscillatory theorems.}

Theorem 4. Let $a>0,0<\beta<1$ such that $\frac{2}{\beta-1}$ is the ratio of two odd integers, $p_{n}>0$, $q_{n}<0$ and the condition

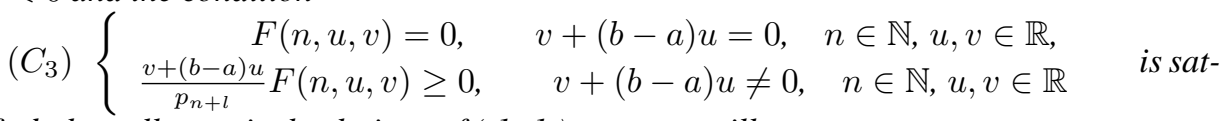

isfied, then all nontrival solutions of ( 1.1 ) are nonoscillatory.

Proof. Let $x$ be any nontrivial solutions of ( 1.1 ). Then $x \in X_{1}$ or $x \in X_{2}$ holds.

(i) If $x \in X_{1}$, then there is some $k \in \mathbb{N}$ such that $\Delta_{l, a} x_{k}=x_{k+l}-a x_{k}=0$. Therefore according to $\left(C_{3}\right), F\left(k, x_{k}, \Delta_{l, b} x_{k}\right)=0$ for some $k \in \mathbb{N}$. Then letting $n=k$ in ( 1 . 1 ), we reach $\Delta_{l, a}\left(p_{k} \Delta_{l, a} x_{k}\right)=0$ and from this we obtain $\Delta_{l, a} x_{k+l}=\frac{a p_{k}}{p_{k+l}} \Delta_{l, a} x_{k}=$ 0 . By repeating this same process, we obtain $\Delta_{l, a} x_{k+2 l}=0$. By induction, we reach $\Delta_{l, a} x_{k+j l}=0$ for some $j \in \mathbb{N}$. Therefore we have

$$
x_{k+j l}=a^{j} x_{k} \text { for some } j \in \mathbb{N} .
$$

Since $a>0$ and $x_{k} \neq 0,\left\{x_{k+j l}\right\}$ is eventually positive or negative according to $x_{k}>$ or $x_{k}<0$.

(ii) If $x \in X_{2}, \frac{v+(b-a) u}{p_{n+l}} F(n, u, v) \geq 0$ holds for $\Delta_{l, a} x_{k} \neq 0$. We aim that $x$ is nonoscillatory. Suppose that $x$ is oscillatory. Thus there are two case;

$\left(c_{1}\right) x_{m}>0, x_{m+l} \leq 0$ or

$\left(c_{2}\right) x_{m} \geq 0, x_{m+l}<0$ for some $m \in \mathbb{N}$. In the case $\left(c_{1}\right)$ we have

$$
\Delta_{l, a} x_{k}<0
$$

We can rewrite the (1.1) in the form (2. 2 ) and by the similar ways (2. 2 )-( 2. 4 ) we obtain

$$
G_{\min }=\left(\frac{\beta-1}{\beta+1}\right) A_{m}\left(\frac{2 A_{m}}{(\beta+1) B_{m}}\right)^{\frac{2}{\beta-1}}>0 \text { at the point } Y_{m}=\left(\frac{2 A_{m}}{(\beta+1) B_{m}}\right)^{\frac{1}{\beta-1}} .
$$


Therefore from ( 2.13$),(2.14)$ and $\left(C_{3}\right)$ we have

$$
\begin{aligned}
\Delta_{l, a} x_{m} \Delta_{l, a} x_{m+l} & \geq\left(\frac{\beta-1}{\beta+1}\right) A_{m}\left(\frac{2 A_{m}}{(\beta+1) B_{m}}\right)^{\frac{2}{\beta-1}} \\
& +\frac{\left(\Delta_{l, b} x_{m}+(b-a) x_{m}\right)}{p_{m+l}} F\left(m, x_{m}, \Delta_{l, b} x_{m}\right) \\
& \geq 0 .
\end{aligned}
$$

Since $X_{2} \ni x_{m}>0$ and $\Delta_{l, a} x_{m} \neq 0$, by (2.13) and (2.15) we have

$$
\Delta_{l, a} x_{m+l} \leq 0 \text {. }
$$

Repeating the same process from ( 2. 13 ) - (2. 15 ) we reach

$$
\Delta_{l, a} x_{m+j l} \leq 0 \text { for all } j \in \mathbb{N} .
$$

Since $a>0$ and $x_{m+l} \leq 0$, we obtain

$$
x_{m+j l} \leq a^{j-1} x_{m+l} \leq 0, \quad j \in \mathbb{N}
$$

This contradicts with to be $x$ is eventually positive.

$\left(c_{2}\right)$ When $x$ is eventually negative, the proof is done in a similar way.

Theorem 5. Let $a>0, \beta>1$ such that $\frac{2}{\beta-1}$ is the ratio of two odd integers, $p_{n}$ and $q_{n}$ are oscillatory with $p_{n} p_{n+l}<0$ and $p_{n} q_{n}<0$, and the condition

$$
\left(C_{4}\right)\left\{\begin{array}{cc}
F(n, u, v)=0, & v+(b-a) u=0, \\
\left(\frac{\beta-1}{\beta+1}\right) \frac{a p_{n}}{p_{n+l}}\left(\frac{2 a p_{n}}{(\beta+1) q_{n}}\right)^{\frac{2}{\beta-1}}+\frac{v+(b-a) u}{p_{n+l}} F(n, u, v) \geq 0, v+(b-a) u \neq 0,
\end{array}\right.
$$

where $n \in \mathbb{N}, u, v \in \mathbb{R}$, is satisfied, then all nontrival solutions of (1.1 ) are nonoscillatory.

Proof. The proof can be made as in Theorem 4.

Theorem 6. Let $a>0,0<\beta<1$ such that $\frac{2}{\beta-1}$ is the ratio of two odd integers, $p_{n}<0$, $q_{n}>0$ and the condition $\left(C_{3}\right)$ is satisfied, then all nontrival solutions of ( 1.1$)$ are nonoscillatory.

Proof. The proof can be made as in Theorem 4.

Remark 4. If $a=b$ conditions $\left(C_{3}\right)$ and $\left(C_{4}\right)$ reduce to

$\left(C_{3}^{*}\right) \frac{v}{p_{n+l}} F(n, u, v) \geq 0$ for $v \neq 0, n \in \mathbb{N}, u, v \in \mathbb{R}$ and

$\left(C_{4}^{*}\right) \quad\left(\frac{\beta-1}{\beta+1}\right) \frac{a p_{n}}{p_{n+l}}\left(\frac{2 a p_{n}}{(\beta+1) q_{n}}\right)^{\frac{2}{\beta-1}}+\frac{v}{p_{n+l}} F(n, u, v) \geq 0$ for $v \neq 0, n \in \mathbb{N}, u, v \in \mathbb{R}$ respectively.

Remark 5. If $b=0$, then ( 1.1 ) becomes the generalized difference equation of the form

$$
\Delta_{l, a}\left(p_{n} \Delta_{l, a} x_{n}\right)+q_{n}\left(\Delta_{l, a} x_{n}\right)^{\beta}=F\left(n, x_{n}, x_{n+l}\right), \quad n \in \mathbb{N},
$$

and for this equation conditions $\left(C_{3}\right)$ and $\left(C_{4}\right)$ reduce to

$$
\begin{aligned}
& \left(C_{3}^{* *}\right) \frac{\left(v^{*}-a u\right)}{p_{n+l}} F\left(n, u, v^{*}\right) \geq 0 \text { for }\left(v^{*}-a u\right) \neq 0, n \in \mathbb{N}, u, v^{*} \in \mathbb{R} \quad \text { and } \\
& \left(C_{4}^{* *}\right)\left(\frac{\beta-1}{\beta+1}\right) \frac{a p_{n}}{p_{n+l}}\left(\frac{2 a p_{n}}{(\beta+1) q_{n}}\right)^{\frac{2}{\beta-1}}+\frac{\left(v^{*}-a u\right)}{p_{n+l}} F\left(n, u, v^{*}\right) \geq 0 \text { for }\left(v^{*}-a u\right) \neq 0, n \in \mathbb{N},
\end{aligned}
$$
$u, v^{*} \in \mathbb{R}$ respectively. 


\subsection{Some examples to nonoscillatory equations.}

Example 5. Consider second order difference equation with generalized difference operators of the form

$$
\Delta_{2,2}^{2} x_{n}-\sqrt[7]{2^{6 n-1}}\left(\Delta_{2,2} x_{n}\right)^{\frac{1}{7}}=x_{n}+\Delta_{2,3} x_{n},
$$

where $a=l=2, p_{n}=1, q_{n}=-\sqrt[7]{2^{6 n-1}}, \beta=\frac{1}{7}, F\left(n, x_{n}, \Delta_{2,3} x_{n}\right)=x_{n}+\Delta_{2,3} x_{n}$. Therefore the second part of condition $\left(C_{3}\right)$ and all assumptions of theorem 4 are satisfied. Then every solution of (2.17) is nonoscillatory. So one of solutions is $x_{n}=2^{n}$.

Example 6. Consider second order difference equation with generalized difference operator of the form

$$
\Delta_{3,2}\left(\left(-\frac{1}{2}\right)^{n} \Delta_{3,2} x_{n}\right)-(-2)^{5 n}\left(\Delta_{3,2} x_{n}\right)^{7}=-\frac{178785135}{6029132}(-1)^{n} x_{n} \Delta_{3,3} x_{n},
$$

where $a=2, b=3, l=3, p_{n}=\left(-\frac{1}{2}\right)^{n}, q_{n}=-(-2)^{5 n}, \beta=7, F\left(n, x_{n}, \Delta_{3,3} x_{n}\right)=$ $-\frac{178785135}{6029132}(-1)^{n} x_{n} \Delta_{3,3} x_{n}$. Therefore the second part of condition $\left(C_{4}\right)$ and all assumptions of theorem 5 are satisfied. Then every solution of (2. 18 ) is nonoscillatory. So one of solutions is $x_{n}=\frac{1}{2^{n}}$.

Example 7. Consider second order difference equation with generalized difference operator of the form

$$
-\Delta_{2,2}^{2} x_{n}+3 \sqrt[3]{4^{(1+n)}}\left(\Delta_{2,2} x_{n}\right)^{\frac{1}{3}}=2 \sqrt{x_{n} \Delta_{2,3} x_{n}}
$$

where $a=2, b=3, l=2, p_{n}=-1, q_{n}=3 \sqrt[3]{4^{(1+n)}}, \beta=\frac{1}{3}, F\left(n, x_{n}, \Delta_{2,3} x_{n}\right)=$ $2 \sqrt{x_{n} \Delta_{2,3} x_{n}}$. Therefore the second part of condition $\left(C_{3}\right)$ and all assumptions of theorem 6 are satisfied. Then every solution of (2. 19$)$ is nonoscillatory. So one of solutions is $x_{n}=2^{n}$.

Remark 6. Examples in 5, 6 and 7 if $l=0, b=0, F(n, u, v)=0 \quad($ or $F(n, u, v)=$ $\left.\lambda(I f-1)^{n}\right)$ are taken, it is seen that the results of Parhi, Popenda and Yang are true.

Conclusion 1. In this manuscript we obtain some new oscilation and nonoscillation criteria for second order nonlinear difference equation with generalized difference operators. It is not possible to decide the oscillatory and nonoscillatory behavior of solutions of ( 2 . 9 )-( 2. 12 ) and (2. 17$)-(2.19)$ by using any of the results reported in [24, 25] and [29]. This implies that the results of our study extend and generalize some known theorems in $[24,25,29]$ and referenced therein.

\section{REFERENCES}

[1] R. P. Agarwal, S. R. Grace and D. O.Regan, Oscillation Theory for Difference an Functional Differential Equations, Kluwer Academic Publishers, Dordrecht (2000).

[2] R. P. Agarwal, Difference Equations and Inequalities, Theory, Methods and Applications. Marcel Dekker, New York (2000).

[3] R. P. Agarwal and P. J. Y. Wong, Advanced Topics in Difference Equations, Kluwer Academic Publishers, Netherlands (1997).

[4] R. Boite and P. Dewilde, Circuit theory and design, North-Holland Publishing Company, Amsterdam (1981).

[5] Y. Bolat and O. Akin, Oscillation criteria for higher order half linear delay difference equations involving generalized difference, Math. Slovaca. 66, No. 3, 1-10 (2016). 
[6] Y. Bolat and O. Akin, A new general forward difference operator and some applications, Turkish Journal of Mathematics and Computer Science, 9 (2018 ) 117-124 ¡https://dergipark.org.tr/tr/pub/tjmcs/issue/42016/468246i

[7] S. Chen and H. Erbe, L, Riccati Techniques and Discrete Oscillations, J. Math. Anal. Appl. 142 $468-487$ (1989).

[8] S. Chen, Disconjugacy, Disfocality, and Oscillation of Second Order Difference Equations, Journal of Differential Equations, 107 383-394 (1994).

[9] J. F. Cheng, YM. Chu, Sufficient and Necessary Conditions for Oscillation of th-Order Differential Equation with Retarded Argument, J Inequal Appl 2009892936 (2009). https://doi.org/10.1155/2009/892936

[10] J. F. Cheng Y. M. Chu, Oscillations of second-order neutral impulsive differential equations, J. Inequal. Appl. 2010 Art. ID 493927, 29 pp.

[11] J. F. Cheng, Y.M. Chu, Oscillatory Criteria for the Two-Dimensional Difference Systems, J Inequal Appl 2010, 209309 (2010). https://doi.org/10.1155/2010/209309

[12] J. F. Cheng and Y. M. Chu, Solution to the Linear Fractional Differential Equation Using Adomian Decomposition Method, Mathematical Problems in Engineering, vol. 2011, Article ID 587068, 14 pages, 2011. https://doi.org/10.1155/2011/587068

[13] J.F. Cheng and Y. M. Chu, Oscillation of one order neutral differential equation with impulses. Commun, Korean Math. Soc. 26, No. 2 (2011) 197-205.

[14] J. F. Cheng and Y. M. Chu, YM, On the Fractional Difference Equations of Order (2, $q)$, Abstract and Applied Analysis, 2011, Article ID 497259, 16 pages, 2011. https://doi.org/10.1155/2011/497259

[15] J. F. Cheng and Y. M. Chu,Fractional Difference Equations with Real Variable, Abstract and Applied Analysis, 2012, Article ID 918529, 24 pages, 2012. https://doi.org/10.1155/2012/918529

[16] S. S. Cheng, T. C. Yan and H. J. Li,Oscillation Criteria for Second Order Difference Equation, Funkcialaj Ekvacioj. 34 (1991) (1991) 223-239.

[17] C. J. Fa. and Y.C. Ming, Necessary and sufficient conditions for oscillation of the solutions of even order differential equations, Rev. Anal. Numér. ThéOr. Approx., 41, No. 1(2012) 18-26. Retrieved from https://ictp.acad.ro/jnaat/journal/article/view/2012-vol41-no1-art2

[18] K. Gopalsamy, Stability and oscillations in population dynamics, Kluwer Academic Publishers, Boston (1992).

[19] X. Z. He, Oscillatory and Asymptotic Behavior of Second Order Nonlinear Difference Equations, J. Math. Anal. Appl. 175, No. 2 (1993) 482-498.

[20] W. G. Kelley, and A. C. Peterson, Difference equations an introduction with applications. Academic Press, Boston (1991).

[21] V. Lakshmikantham and D. Trigiante, Theory of difference equations, numerical methods and applications, Academic Press, New York (1988).

[22] C. A. Marinov and P. Neittaanmaki, Mathematical models in electrical circuits: theory applications, Kluwer, Dordrecht (1991).

[23] V. L. Nagy and D. L. Powers, On rectangular systems of differential equations and their application to circuit theory Journal of the Franklin Institute, 299, No. 6 (1975) 399-407.

[24] N. Parhi, Oscillation and non-oscillation of solutions of second order difference equations involving generalized difference, Applied Mathematics and Computation, 218 (2011) 458-468.

[25] J. Popenda, Oscillation and nonoscillation theorems for second order difference equations, J. Math. Anal. Appl. 123 (1987) 34-38.

[26] S. S. Santra, O. Bazighifan, H. Ahmad and Y. M. Chu, Second-Order Differential Equation: Oscillation Theorems and Applications, Mathematical Problems in Engineering, 2020, Article ID 8820066, 6 pages, 2020. https://doi.org/10.1155/2020/8820066

[27] Z. Szafranski and B. Szmanda, Oscillatory Behavior of Difference Equations of Second Order, J. Math. Anal. Appl. 150 (1990) 414-424.

[28] B. Szmanda, Nonoscillation, Oscillation and Growth of Solutions of Nonlinear Difference Equations of Second Order, J. Math. Anal. Appl.109 (1985) 22-30.

[29] M. C. Tan, and E. H. Yang, Oscillation and nonoscillation theorems for second order difference equations. J. Math. Anal. Appl. 276 (2002) 239-247.

[30] E. Thandapani and B. S. Lalli, Oscillation criteria for a second order damped difference equation, Applied Mathematics Letters, 8, No. 1 (1995) 1-6. 
[31] E. Thandapani, Oscillation theorems for a second order damped nonlinear difference equation, Czechoslovak Mathematical Journal, 45, No. 120 (1995) 327-335.

[32] P. J. Y. Wong, and R. P. Agarwal, Oscillation theorems and existence of positive monotone solutions for second order nonlinear difference equations, Mathematical and Computer Modelling, 21, No.3 (1995) 6384.

[33] B.G. Zhang, Oscillation and Asymptotic behavior of Second Order Difference Equations, J. Math. Anal. Appl. 173, No. 1 (1993) 58-68.

[34] Zhang, BG, Chen, GD: Oscillation of Certain Second Order Nonlinear Difference Equations. J. Math. Anal. Appl. 199 (1996) 827-841.

[35] B. G. Zhang and Y. Zhou, Oscillation and Nonoscillation for Second-Order Linear Difference Equations, Computers and Mathematics with Applications, 39 (2000)1-7.

[36] Q. Zhang, XJ. Wei, Stability analysis for cellular neural networks with variable delays, Chaos, Solitons \& Fractals. 28 (2006) 331-336.

[37] J. Zhou, T. Chen, and L. R. Xiang, Synchronization of delayed neural networks based on adaptive control and parameters identification, Chaos, Solitons \& Fractals. 27 (2006) 905-913. 